\title{
Systems of coupled Poisson equations with critical growth in unbounded domains*
}

\author{
Fabrice Colin ${ }^{\dagger} \quad$ Marlène Frigon ${ }^{\ddagger}$
}

CRM-2933

June 2003

\footnotetext{
*This work was partially supported by CRSNG Canada and FCAR Québec

${ }^{\dagger}$ Département de mathématiques et d'informatique, Université de Sherbrooke, Sherbrooke, QC J1K 2R1, Canada; fabrice.colin@dmi.usherb.ca

${ }^{\ddagger}$ Département de mathématiques et de statistique, Université de Montréal, CP 6128, succ. Centre-ville, Montréal, QC H3C 3J7, Canada, frigon@dms.umontreal.ca
} 



\begin{abstract}
We establish the existence of a nontrivial solution to systems of coupled Poisson equations with critical growth in unbounded domains. The proofs rely on a generalized linking theorem due to Krysewski and Szulkin [9], and on a concentration-compactness argument since the Palais-Smale condition fails at all critical levels.
\end{abstract}

Mathematical Subject Classification. 35J50, 35J55

Keywords and Phrases. Coupled Poisson equations, critical exponant, unbounded domains, variational methods, generalized linking theorem 



\section{Introduction}

In this paper, we consider the following systems of coupled Poisson equations with critical growth in unbounded domains

$$
\begin{aligned}
& -\Delta u=|v|^{2^{*}-2} v \\
& -\Delta v=|u|^{2^{*}-2} u \\
& u, v \in D_{0}^{1,2}\left(\mathbb{R}^{N} \backslash \mathbb{Z}^{N}\right) ;
\end{aligned}
$$

and

$$
\begin{gathered}
-\Delta u=\gamma v+|v|^{2^{*}-2} v \\
-\Delta v=\lambda u+|u|^{2^{*}-2} u \\
u, v \in H_{0}^{1}(\Omega),
\end{gathered}
$$

where $\Omega:=\mathbb{R}^{l} \times \omega$ is a cylindrical domain with $\omega$ a bounded domain of $\mathbb{R}^{N-l}$ for $1 \leq l \leq N-1$, and $0<\gamma, \lambda<$ $\lambda_{1}(\Omega)$, with $\lambda_{1}(\Omega)$ the best constant in the Poincaré inequality. Those problems have a variational formulation with respectively the functionals:

$$
\begin{aligned}
\varphi(u, v) & :=\int_{\Omega} \nabla u \cdot \nabla v-\frac{|u|^{2^{*}}}{2^{*}}-\frac{|v|^{2^{*}}}{2^{*}} d x \\
\varphi_{1}(u, v) & :=\int_{\Omega} \nabla u \cdot \nabla v-\frac{\gamma u^{2}}{2}-\frac{\lambda v^{2}}{2}-\frac{|u|^{2^{*}}}{2^{*}}-\frac{|v|^{2^{*}}}{2^{*}} d x
\end{aligned}
$$

Observe that the first part is a strongly indefinite operator. In particular, those functionals have the form

$$
\frac{\|Q(u, v)\|^{2}}{2}-\frac{\|P(u, v)\|^{2}}{2}-\psi(u, v)
$$

where the Hilbert space $X=Y \oplus Z$ with $Y$ and $Z$ infinite dimensional subspaces, and $P$ and $Q$ are respectively the orthogonal projections on $Y$ and $Z$. Therefore, the classical min-max results can not be applied. In particular, since $\varphi$ and $\varphi_{1}$ are invariant under $\mathbb{Z}^{N}$-translations and $\mathbb{R}^{l}$-translations respectively, the Palais-Smale condition fails at all critical levels.

This type of problems on bounded domains were studied in the subcritical growth case by Husholf and van der Vorst [7] in using the Indefinite Functional Theorem due to Benci and Rabinowitz [1]; and by Felmer and Wang [6] who obtained multiplicity results in using Galerkin type methods. The critical growth case was studied by Husholf, Mitidieri and van der Vorst [8] where they used a dual formulation due to Clarke and Ekeland [2].

Here, we will use concentration-compactness techniques and we will applied a result obtained by Krysewski and Szulkin [9] in 1996, which is a generalized linking theorem for a suitable functional defined on a Hilbert space $X=Y \oplus Z$ with $Y$ a separable subspace of $X$ which could be infinite dimensional and $Z:=Y^{\perp}$. In their proof, they considered another topology on $Y$ and they introduced a generalized degree theory. We state a corollary of their result that will be used in what follows (see also [13]).

Let $P: X \rightarrow Y, Q: X \rightarrow Z$ be the orthogonal projections. Now, let $\rho>r>0$ and let $z \in Z$ be such that $\|z\|=1$. Define

$$
\begin{aligned}
M & :=\{u=y+\lambda z:\|u\| \leq \rho, \lambda \geq 0, y \in Y\}, \\
M_{0} & :=\{u=y+\lambda z: y \in Y,\|u\|=\rho \text { and } \lambda \geq 0 \text { or }\|u\| \leq \rho \text { and } \lambda=0\}, \\
N & :=\{u \in Z:\|u\|=\rho\} .
\end{aligned}
$$

Theorem 1.1. (Kryszewski-Szulkin, 1996). Let $\psi \in C^{1}(X, \mathbb{R})$ be weakly sequentially lower semicontinuous, bounded below and such that $\psi^{\prime}$ is weakly sequentially continuous. If

$$
\varphi(u):=\frac{\|Q u\|^{2}}{2}-\frac{\|P u\|^{2}}{2}-\psi(u)
$$

satisfies

$$
b:=\inf _{N} \varphi>0=\sup _{M_{0}} \varphi, \quad d:=\sup _{M} \varphi<\infty,
$$

then there exists $c \in[b, d]$ and a sequence $\left(u_{n}\right) \subset X$ such that

$$
\varphi\left(u_{n}\right) \rightarrow c, \quad \varphi^{\prime}\left(u_{n}\right) \rightarrow 0 .
$$




\section{Preliminaries}

In the sequel $N \geq 3$ and $2^{*}:=2 N /(N-2)$. We consider the Hilbert space

$$
D^{1,2}\left(\mathbb{R}^{N}\right):=\left\{u \in L^{2^{*}}\left(\mathbb{R}^{N}\right): \nabla u \in L^{2}\left(\mathbb{R}^{N}\right)\right\},
$$

endowed with the inner product

$$
\int_{\mathbb{R}^{N}} \nabla u(x) \cdot \nabla v(x) d x
$$

and the associated norm noted $\|u\|$. The Sobolev imbedding Theorem asserts that the imbedding

$$
D^{1,2}\left(\mathbb{R}^{N}\right) \hookrightarrow L^{2^{*}}\left(\mathbb{R}^{N}\right)
$$

is continuous.

For a domain $\Omega \subset \mathbb{R}^{N}$, we denote by $D_{0}^{1,2}(\Omega)$ the closure of $D(\Omega)$ in $D^{1,2}\left(\mathbb{R}^{N}\right)$. Obviously $D^{1,2}\left(\mathbb{R}^{N}\right)=D_{0}^{1,2}\left(\mathbb{R}^{N}\right)$, and when the Poincaré inequality is satisfied, $D_{0}^{1,2}(\Omega)=H_{0}^{1}(\Omega)$.

Let us recall that the embedding $D^{1,2}\left(\mathbb{R}^{N}\right) \hookrightarrow L^{2^{*}}\left(\mathbb{R}^{N}\right)$ is not compact, but we have the following well known result, see Wang and Willem [12] for a generalization.

Lemma 2.1. If $u_{n} \rightarrow u$ in $D^{1,2}\left(\mathbb{R}^{N}\right)$, then $u_{n} \rightarrow u$ in $L_{l o c}^{2}\left(\mathbb{R}^{N}\right)$.

The following lemma due to Ramos, Wang and Willem [11] gives sufficient conditions to ensure the convergence to 0 in $L^{2^{*}}\left(\mathbb{R}^{N}\right)$ of a sequence in $H^{1}\left(\mathbb{R}^{N}\right)$. This type of results was firstly established by P. L. Lions [10] for an exponent $p<2^{*}$. See also Colin [3] or [4] for a similar result in weighted spaces on a cylindrical domain.

Lemma 2.2. Let $r>0$. If $\left(u_{n}\right)$ is bounded in $H^{1}\left(\mathbb{R}^{N}\right)$ and if

$$
\sup _{x \in \mathbb{R}^{N}} \int_{B(x, r)}\left|u_{n}\right|^{2^{*}} d x \rightarrow 0 \quad \text { as } n \rightarrow \infty
$$

then $u_{n} \rightarrow 0$ in $L^{2^{*}}\left(\mathbb{R}^{N}\right)$.

Here, we establish an analogous result in $D_{0}^{1,2}\left(\mathbb{R}^{N} \backslash \mathbb{Z}^{N}\right)$. The choice of the domain $\mathbb{R}^{N} \backslash \mathbb{Z}^{N}$ could seem surprising but it is, in some sense, the biggest domain in which we are able to find a weak solution using our method. If a more regular domain is needed, notice that all the following results are also true for domains of the form:

$$
\mathbb{R}^{N} \backslash \bigcup_{a \in \mathbb{Z}^{N}} \overline{\tau_{a}(\omega)}
$$

where $\tau_{a}(x)=x+a$ is a translation applied to a domain $\omega$ such that $0 \in \omega \subset B(0, R), R<1 / 2$ and such that $\partial \omega$ is of class $C^{1}$. Indeed, these domains are invariant under $\mathbb{Z}^{N}$-translations and have a $C^{1}$-boundary.

Lemma 2.3. Let $\left(u_{n}\right) \subset D_{0}^{1,2}\left(\mathbb{R}^{N} \backslash \mathbb{Z}^{N}\right)$ be a bounded sequence. If

$$
\sup _{a \in \mathbb{Z}^{N}} \int_{B(a, \sqrt{N})}\left|u_{n}\right|^{2^{*}} \rightarrow 0, \quad \text { when } n \rightarrow \infty
$$

then $u_{n} \rightarrow 0$ in $L^{2^{*}}\left(\mathbb{R}^{N}\right)$.

In order to prove this result we will need the following notations and results. Let $\Omega:=\mathbb{R}^{l} \times \omega$, where $\omega$ is a domain of $\mathbb{R}^{N-l}$ with $l \in\{1, \ldots, N-1\}$. Let us denote

$$
\left.\Omega_{+}:=\right] 0, \infty\left[{ }^{l} \times \omega,\right.
$$

and for each function $u \in C^{\infty}\left(\Omega_{+}\right)$, we define its symmetric extension $u^{*}$ on $\Omega$, and for each $i$ such that $1 \leq i \leq l$, an another extension $u^{\square i}$ by:

$$
\begin{gathered}
u^{*}(x)=u^{*}\left(y_{1}, \ldots, y_{l}, z_{l+1}, \ldots, z_{N}\right)= \begin{cases}u(x) & \text { if } x \in \Omega_{+}, \\
u\left(\left|y_{1}\right|, \ldots,\left|y_{l}\right|, z_{l+1}, \ldots, z_{N}\right) & \text { otherwise, }\end{cases} \\
u^{\square i}(x)=u^{\square i}\left(y_{1}, \ldots, y_{l}, z_{l+1}, \ldots, z_{N}\right)= \begin{cases}u\left(\left|y_{1}\right|, \ldots,\left|y_{l}\right|, z_{l+1}, \ldots, z_{N}\right) & \text { if } y_{i}>0, \\
-u\left(\left|y_{1}\right|, \ldots,\left|y_{l}\right|, z_{l+1}, \ldots, z_{N}\right) & \text { if } y_{i}<0,\end{cases}
\end{gathered}
$$

where $x=(y, z)$, with $y \in \mathbb{R}^{l}$ and $z \in \omega$. 
Proposition 2.4. (Symmetric extension) Let $\Omega$ be as above and $1 \leq p<\infty$. If $u \in D(\Omega)$ and in choosing to denote $u_{+}:=\left.u\right|_{\Omega_{+}}$, then $u_{+}^{*} \in W^{1, p}(\Omega)$ and more precisely we have the following equalities:

$$
\begin{aligned}
& \frac{\partial u_{+}^{*}}{\partial y_{i}}=\left(\frac{\partial u}{\partial y_{i}}\right)^{\square i} \quad \text { where } 1 \leq i \leq l, \\
& \frac{\partial u_{+}^{*}}{\partial z_{j}}=\left(\frac{\partial u}{\partial z_{j}}\right)^{*} \quad \text { where } l+1 \leq j \leq N .
\end{aligned}
$$

Proposition 2.5. (Chain rule) Let $U$ and $U^{\prime}$ be two open sets of $\mathbb{R}^{N}, p \geq 1$ and $H: U^{\prime} \rightarrow U$ a bijective application such that

$$
H \in C^{1}\left(U^{\prime}\right), \quad H^{-1} \in C^{1}(U), \quad J a c H \in L^{\infty}\left(U^{\prime}\right), \quad J a c H^{-1} \in L^{\infty}(U),
$$

where JacH denote the Jacobian matrix. Let $u \in W^{1, p}(U)$, then $u \circ H \in W^{1, p}\left(U^{\prime}\right)$ and, in addition,

$$
(u \circ H)^{\prime}(y)=u^{\prime}(H(y)) \circ H^{\prime}(y) .
$$

Proof of Lemma 2.3. Since the problem is invariant by $\mathbb{Z}^{N}$ translations, we may assume for now that $a=0$. Let us consider $u \in D\left(\mathbb{R}^{N} \backslash \mathbb{Z}^{N}\right)$ and let $\Psi \in D\left(\mathbb{R}^{N}\right)$ be such that $0 \leq \Psi \leq 1$ and

$$
\Psi(x):= \begin{cases}1 & \text { if } x \in B(0, \sqrt{N}) \\ 0 & \text { if }|x|>\sqrt{N}+1\end{cases}
$$

The Sobolev imbedding Theorem implies that

$$
\left(\int_{B(0, \sqrt{N})}|u|^{2^{*}} d x\right)^{2 / 2^{*}} \leq C \int_{B(0, \sqrt{N}+1)}|\nabla(\Psi u)|^{2} d x .
$$

In order to bound $\int|\nabla(\Psi u)|^{2}$ by $\int|\nabla u|^{2}$, we have to consider the following decomposition

$$
\begin{aligned}
\int_{B(0, \sqrt{N}+1)}|\nabla(\Psi u)|^{2} d x= & \int_{B(0, \sqrt{N}+1)} \Psi^{2}|\nabla u|^{2} d x+\int_{B(0, \sqrt{N}+1)} u^{2}|\nabla \Psi|^{2} d x \\
& +2 \int_{B(0, \sqrt{N}+1)} u \Psi(\nabla u \cdot \nabla \Psi) .
\end{aligned}
$$

Working on the third term, we obtain:

$$
\begin{aligned}
\int_{B(0, \sqrt{N}+1)} u \Psi(\nabla u \cdot \nabla \Psi) d x & =\frac{1}{2} \int_{B(0, \sqrt{N}+1)}\left(\nabla u^{2} \cdot \Psi \nabla \Psi\right) d x \\
& =-\frac{1}{2} \int_{B(0, \sqrt{N}+1)} u^{2} \operatorname{div}(\Psi \nabla \Psi) d x \\
& \leq C \int_{B(0, \sqrt{N}+1)} u^{2} d x .
\end{aligned}
$$

Please note that to avoid the use of too many different letters, we will denote by $C$ all real positive constants.

In a next step, we have to bound

$$
\int_{B(0, \sqrt{N}+1)} u^{2} d x \quad \text { by } \quad \int_{B(0, \sqrt{N}+1)}|\nabla u|^{2} d x .
$$

For $r>0$, denote by $P_{r}$ the polar coordinates transformation

$$
\left.P_{r}:\right] 0, r(\sqrt{N}+1)[\times] 0,2 \pi[\times]-\frac{\pi}{2}, \frac{\pi}{2}\left[^{N-2} \mapsto B(0, r(\sqrt{N}+1)) \backslash \mathfrak{N}_{r},\right.
$$

where $\mathfrak{N}_{r}$ is a null set. It is also clear that $\mathrm{Jac} P_{r}$ is bounded. Lemmas 2.4 and 2.5 imply that we can extend $u \circ P_{1}$ with respect to the first variable in order to obtain the symmetric extension $\left(u \circ P_{1}\right)^{*}$ over the new domain:

$$
] 0,2 \sqrt{N}+2[\times] 0,2 \pi[\times]-\frac{\pi}{2}, \frac{\pi}{2}\left[^{N-2} .\right.
$$


It follows that $u^{*}:=\left(u \circ P_{1}\right)^{*} \circ P_{2}^{-1}$ is defined on the ball $B(0,2 \sqrt{N}+2)$ but a null set, and $u^{*} \in D_{0}^{1,2}(B(0,2 \sqrt{N}+2))$ which is an extension of $u$ having, in addition, the following property:

$$
\int_{B(0,2 \sqrt{N}+2)}\left|\nabla u^{*}\right|^{2} d x \leq C \int_{B(0, \sqrt{N}+1)}|\nabla u|^{2} d x .
$$

This, the fact that $u=u^{*}$ on $B(0, \sqrt{N}+1)$, and the Poincaré inequality applied to the function $u^{*}$ lead to

$$
\int_{B(0, \sqrt{N}+1)} u^{2} d x \leq C \int_{B(0, \sqrt{N}+1)}|\nabla u|^{2} d x .
$$

In combining (2.1)-(2.4) and in using the $\mathbb{Z}^{N}$ invariance, we obtain for every $a \in \mathbb{Z}^{N}$ and for every $u \in D_{0}^{1,2}\left(\mathbb{R}^{N} \backslash\right.$ $\left.\mathbb{Z}^{N}\right)$, by a density argument

$$
\int_{B(a, \sqrt{N}+1)}|u|^{2^{*}} d x \leq C\left(\int_{B(a, \sqrt{N}+1)}|\nabla u|^{2} d x\right)^{2^{*} / 2}
$$

and hence, for any $\lambda>0$,

$$
\int_{B(a, \sqrt{N})}|u|^{2^{*}} d x \leq C^{\lambda}\left(\int_{B(a, \sqrt{N}+1)}|\nabla u|^{2} d x\right)^{\left(2^{*} / 2\right) \lambda}\left(\int_{B(a, \sqrt{N})}|u|^{2^{*}} d x\right)^{1-\lambda} .
$$

In choosing $\lambda=2 / 2^{*}$ we may write, since every $x \in \mathbb{R}$ is contained in at most $(\sqrt{N})^{N}$ balls,

$$
\int_{\mathbb{R}^{N}}|u|^{2^{*}} d x \leq C\left(\int_{\mathbb{R}^{N}}|\nabla u|^{2} d x\right) \sup _{a \in \mathbb{Z}^{N}}\left(\int_{B(a, \sqrt{N})}|u|^{2^{*}} d x\right)^{\left(2^{*}-2\right) / 2^{*}} .
$$

Under the assumptions of the lemma, we conclude that $u_{n} \rightarrow 0$ in $L^{2^{*}}\left(\mathbb{R}^{N}\right)$.

\section{Applications}

In this section, we start in presenting an application to the following system of two coupled Poisson equations:

$$
\left\{\begin{aligned}
-\Delta u & =|v|^{2^{*}-2} v \\
-\Delta v & =|u|^{2^{*}-2} u \\
u, v & \in D_{0}^{1,2}\left(\mathbb{R}^{N} \backslash \mathbb{Z}^{N}\right)
\end{aligned}\right.
$$

First of all, we establish some general results. Let $\Omega$ be a domain in $\mathbb{R}^{N}$. Denote $X:=D_{0}^{1,2}(\Omega) \times D_{0}^{1,2}(\Omega)$ the Hilbert space endowed with the scalar product:

$$
\left\langle(u, v),\left(u_{1}, v_{1}\right)\right\rangle:=\int_{\Omega} \nabla u \cdot \nabla u_{1}+\nabla v \cdot \nabla v_{1} d x,
$$

If we set

$$
Y:=\{(-v, v) \in X\} \quad \text { and } \quad Z:=\{(u, u) \in X\},
$$

it is easy to check that $X=Y \oplus Z$ since

$$
(u, v)=\frac{1}{2}(u+v, u+v)+\frac{1}{2}(-v+u, v-u) .
$$

Let us denote by $P$ (resp. $Q$ ) the projection of $X$ onto $Y$ (resp. $Z$ ).

Define the functional $\varphi: X \rightarrow \mathbb{R}$ by

$$
\begin{aligned}
\varphi(u, v) & :=\int_{\Omega} \nabla u \cdot \nabla v-\frac{|u|^{2^{*}}}{2^{*}}-\frac{|v|^{2^{*}}}{2^{*}} d x \\
& =\frac{\|Q(u, v)\|^{2}}{2}-\frac{\|P(u, v)\|^{2}}{2}-\psi(u, v),
\end{aligned}
$$

where

$$
\psi(u, v):=\int_{\Omega} \frac{|u|^{2^{*}}}{2^{*}}+\frac{|v|^{2^{*}}}{2^{*}} d x
$$

Observe that the problem (3.1) allows a variationnal formulation since its solutions are critical points of $\varphi$ for $\Omega=\mathbb{R}^{N} \backslash \mathbb{Z}^{N}$. 
Lemma 3.1. The function $\psi$ is $C^{1}$ and weakly sequentially lower semi-continuous. Moreover, for every $(u, v),(w, z) \in$ $X$,

$$
\left\langle\varphi^{\prime}(u, v),(w, z)\right\rangle=\int_{\Omega} \nabla u \cdot \nabla z+\nabla v \cdot \nabla w-|u|^{2^{*}-2} u w-|v|^{2^{*}-2} v z d x .
$$

Proof. It is clear that $\psi$ is weakly sequentially lower semi-continuous since the embedding $X \hookrightarrow L^{2^{*}}(\Omega) \times L^{2^{*}}(\Omega)$ is linear continuous and the norm on $L^{2^{*}}(\Omega)$ is weakly sequentially continuous.

Let $(u, v),(w, z) \in X$. For $x \in \Omega$ and $|t| \in] 0,1[$, there exists $\lambda \in] 0,1[$ such that

$$
\begin{aligned}
\frac{|| u(x)+\left.t w(x)\right|^{2^{*}}-|u(x)|^{2^{*}} \mid}{2^{*}|t|} & =|| u(x)+\left.\lambda t w(x)\right|^{2^{*}-2}(u(x)+\lambda t w(x)) w(x) \mid \\
& =|u(x)+\lambda t w(x)|^{2^{*}-1}|w(x)| \\
& \leq(|u(x)|+|w(x)|)^{2^{*}-1}|w(x)| .
\end{aligned}
$$

The term on the right hand side is in $L^{1}$ by the Hölder inequality. The Lebesgue dominated convergence Theorem implies that

$$
\lim _{t \rightarrow 0} \frac{1}{2^{*}|t|} \int_{\Omega}|u(x)+t w(x)|^{2^{*}}-|u(x)|^{2^{*}} d x=\int_{\Omega}|u(x)|^{2^{*}-2} u(x) w(x) d x .
$$

Similarly,

$$
\lim _{t \rightarrow 0} \frac{1}{2^{*}|t|} \int_{\Omega}|v(x)+t z(x)|^{2^{*}}-|v(x)|^{2^{*}} d x=\int_{\Omega}|v(x)|^{2^{*}-2} v(x) z(x) d x .
$$

Now, assume that $\left(u_{n}, v_{n}\right) \rightarrow(u, v)$ in $X$. From the continuous embedding $D_{0}^{1,2}(\Omega) \hookrightarrow L^{2^{*}}(\Omega)$, we deduce that

$$
\left(\left|u_{n}\right|^{2^{*}-2} u_{n},\left|v_{n}\right|^{2^{*}-2} v_{n}\right) \rightarrow\left(|u|^{2^{*}-2} u,|v|^{2^{*}-2} v\right) \quad \text { in } L^{2^{*} /\left(2^{*}-1\right)} \times L^{2^{*} /\left(2^{*}-1\right)},
$$

and,

$$
\begin{aligned}
& \left.\left|\int_{\Omega}\right| u_{n}(x)\right|^{2^{*}-2} u_{n}(x) w(x)-|u(x)|^{2^{*}-2} u(x) w(x) \\
& \quad+\left|v_{n}(x)\right|^{2^{*}-2} v_{n}(x) z(x)-|v(x)|^{2^{*}-2} v(x) z(x) d x \mid \\
& \leq|| w \|_{L^{2^{*}}}\left(\left.\int_{\Omega}|| u_{n}(x)\right|^{2^{*}-2} u_{n}(x)-\left.|u(x)|^{2^{*}-2} u(x)\right|^{2^{*} / 2^{*}-1} d x\right)^{\left(2^{*}-1\right) / 2^{*}} \\
& \quad+\|z\|_{L^{2^{*}}}\left(\left.\int_{\Omega}|| v_{n}(x)\right|^{2^{*}-2} v_{n}(x)-\left.|v(x)|^{2^{*}-2} v(x)\right|^{2^{*} / 2^{*}-1} d x\right)^{\left(2^{*}-1\right) / 2^{*}} \\
& \leq \\
& \quad k\left(\left(\left.\int_{\Omega}|| u_{n}(x)\right|^{2^{*}-2} u_{n}(x)-\left.|u(x)|^{2^{*}-2} u(x)\right|^{2^{*} / 2^{*}-1} d x\right)^{\left(2^{*}-1\right) / 2^{*}}\right. \\
& \left.\quad+\left(\left.\int_{\Omega}|| v_{n}(x)\right|^{2^{*}-2} v_{n}(x)-\left.|v(x)|^{2^{*}-2} v(x)\right|^{2^{*} / 2^{*}-1} d x\right)^{\left(2^{*}-1\right) / 2^{*}}\right),
\end{aligned}
$$

for every $(w, z) \in X$ such that $\|(w, z)\| \leq 1$. This shows that the Gâteau derivative of $\psi$ is continuous and hence $\psi$ is $C^{1}$.

On the other hand,

$$
\lim _{t \rightarrow 0} \frac{1}{|t|} \int_{\Omega} \nabla(u+t w) \cdot \nabla(v+t z)-\nabla u \cdot \nabla v d x=\langle(u, v),(z, w)\rangle .
$$

This Gâteau derivative is obviously continuous; so $\varphi$ is $C^{1}$ and

$$
\left\langle\varphi^{\prime}(u, v),(w, z)\right\rangle=\int_{\Omega} \nabla u \cdot \nabla z+\nabla v \cdot \nabla w-|u|^{2^{*}-2} u w-|v|^{2^{*}-2} v z d x .
$$

Lemma 3.2. The map $\psi^{\prime}$ is weakly sequentially continuous. 
Proof. Suppose that $\left(u_{n}, v_{n}\right) \rightarrow(u, v)$ in $X$. So, $\left\{u_{n}\right\}$ and $\left\{v_{n}\right\}$ are bounded in $L^{2^{*}}(\Omega)$ and hence $\left\{\left|u_{n}\right|^{2^{*}-2} u_{n}\right\}$ and $\left\{\left|v_{n}\right|^{2^{*}-2} v_{n}\right\}$ are bounded in $\left(L^{2^{*}}(\Omega)\right)^{\prime}$. Lemma 2.1 implies that $u_{n} \rightarrow u$ and $v_{n} \rightarrow v$ in $L_{l o c}^{2}(\Omega)$. Therefore, for every $w, z \in D(\Omega)$,

$$
\int_{\Omega}\left|u_{n}\right|^{2^{*}-2} u_{n} w+\left|v_{n}\right|^{2^{*}-2} v_{n} z d x \rightarrow \int_{\Omega}|u|^{2^{*}-2} u w+|v|^{2^{*}-2} v z d x
$$

i.e. $\left\langle\psi^{\prime}\left(u_{n}, v_{n}\right),(w, z)\right\rangle \rightarrow\left\langle\psi^{\prime}(u, v),(w, z)\right\rangle$. Moreover, $\left\{\psi^{\prime}\left(u_{n}, v_{n}\right)\right\}$ is bounded in $X$, so $\psi^{\prime}\left(u_{n}, v_{n}\right) \rightarrow \psi^{\prime}(u, v)$.

Now, we want to show that $\varphi$ satisfies (1.4). Fix $(z, z) \in Z$ such that $\|(z, z)\|=1$.

Lemma 3.3. There exists $r>0$ such that

$$
b:=\inf _{\substack{(u, u) \in Z \\\|(u, u)\|=r}} \varphi(u, u)>0=\min _{\substack{(u, u) \in Z \\\|(u, u)\| \leq r}} \varphi(u, u) .
$$

Moreover, there exists $\rho>r$ such that

$$
\max _{M_{0}} \varphi=0 \quad \text { and } \quad d:=\sup _{M} \varphi<\infty,
$$

where $M$ and $M_{0}$ are given respectively by (1.1) and (1.2).

Proof. The Sobolev embedding Theorem of $D_{0}^{1,2}(\Omega) \hookrightarrow L^{2^{*}}(\Omega)$ implies directly (3.4) since for $(u, u) \in Z$,

$$
\varphi(u, u) \geq \frac{\|(u, u)\|^{2}}{2}-C\|(u, u)\|^{2^{*}} .
$$

Observe that on $Y$, we have

$$
\varphi(-v, v)=\frac{-\|(-v, v)\|^{2}}{2}-\frac{2}{2^{*}} \int_{\Omega}|v|^{2^{*}} d x \leq 0 .
$$

Denote by $W$ the closure of $Y \oplus \mathbb{R}(z, z)$ in $L^{2^{*}}(\Omega) \times L^{2^{*}}(\Omega)$. Since there exists a continuous projection of $W$ onto $\mathbb{R}(z, z)$ and all the norms are equivalent on the latter space, we have

$$
\begin{aligned}
\varphi((-v, v)+\lambda(z, z))= & -\frac{1}{2}\|(-v, v)\|^{2}+\frac{\lambda^{2}}{2}\|(z, z)\|^{2} \\
& -\frac{1}{2^{*}}\left(|-v+\lambda z|_{2^{*}}^{2^{*}}+|v+\lambda z|_{2^{*}}^{2^{*}}\right) \\
\leq & -\frac{1}{2}\|(-v, v)\|^{2}+\frac{\lambda^{2}}{2}-C \lambda^{2^{*}} .
\end{aligned}
$$

It follows that for $w \in Y \oplus \mathbb{R}(z, z)$

$$
\varphi(w) \rightarrow-\infty \quad \text { whenever }\|w\| \rightarrow \infty
$$

and so, for some $\rho>r, \max _{M_{0}} \varphi=0$.

Finally, the Cauchy-Schwarz inequality and the Sobolev inequality imply that $\varphi$ maps bounded sets into bounded sets, hence $\sup _{M} \varphi<\infty$.

Lemma 3.4. There exists $c \in[b, d]$ and a bounded sequence $\left\{\left(u_{n}, v_{n}\right)\right\}$ in $X$ such that

$$
\varphi\left(u_{n}, v_{n}\right) \rightarrow c>0, \quad \varphi^{\prime}\left(u_{n}, v_{n}\right) \rightarrow 0 .
$$

Proof. It follows from Theorem 1.1 and Lemmas 3.1-3.3 that there exist $c \in[b, d]$ and a sequence $\left\{\left(u_{n}, v_{n}\right)\right\}$ in $X$ satisfying (3.6).

Observe that

$$
\begin{aligned}
\varphi\left(u_{n}, v_{n}\right)-\frac{1}{2}\left\langle\varphi^{\prime}\left(u_{n}, v_{n}\right),\left(u_{n}, v_{n}\right)\right\rangle= & \int_{\Omega} \nabla u_{n} \cdot \nabla v_{n}-\frac{\left|u_{n}\right|^{2^{*}}}{2^{*}}-\frac{\left|v_{n}\right|^{2^{*}}}{2^{*}} \\
& -\frac{1}{2}\left(2 \nabla u_{n} \cdot \nabla v_{n}-\left|u_{n}\right|^{2^{*}}-\left|v_{n}\right|^{2^{*}}\right) \\
= & \mu\left(\left\|u_{n}\right\|_{L^{2^{*}}}^{2^{*}}+\left\|v_{n}\right\|_{L^{2^{*}}}^{2^{*}}\right),
\end{aligned}
$$


with $\mu=1 / 2-1 / 2^{*}$. So, for $\varepsilon>0$, and $n \in \mathbb{N}$ large enough,

$$
c+\varepsilon+\varepsilon\left\|\left(u_{n}, v_{n}\right)\right\| \geq \mu\left(\left\|u_{n}\right\|_{L^{2^{*}}}^{2^{*}}+\left\|v_{n}\right\|_{L^{2^{*}}}^{2^{*}}\right) \geq c-\varepsilon-\varepsilon\left\|\left(u_{n}, v_{n}\right)\right\| .
$$

On the other hand,

$$
\begin{aligned}
\left\|Q\left(u_{n}, v_{n}\right)\right\|^{2}-\varepsilon & \left\|Q\left(u_{n}, v_{n}\right)\right\| \\
& \leq\left|\left\|Q\left(u_{n}, v_{n}\right)\right\|^{2}-\frac{1}{2}\left\langle\varphi^{\prime}\left(u_{n}, v_{n}\right),\left(u_{n}+v_{n}, u_{n}+v_{n}\right)\right\rangle\right| \\
& =\int_{\Omega}\left(\left|u_{n}\right|^{2^{*}-2} u_{n}+\left|v_{n}\right|^{2^{*}-2} v_{n}\right)\left(\frac{u_{n}+v_{n}}{2}\right) \\
& \leq\left\|Q\left(u_{n}, v_{n}\right)\right\|_{L^{2^{*}} \times L^{2^{*}}}\left(\left\|u_{n}\right\|_{L^{2^{*}}}^{2^{*}-1}+\left\|v_{n}\right\|_{L^{2^{*}}}^{2^{*}-1}\right) \\
& \leq k\left\|Q\left(u_{n}, v_{n}\right)\right\|\left(\left\|u_{n}\right\|_{L^{2^{*}}}^{2^{*}-1}+\left\|v_{n}\right\|_{L^{2^{*}}}^{2^{*}-1}\right) .
\end{aligned}
$$

Similarly,

$$
\begin{aligned}
\left\|P\left(u_{n}, v_{n}\right)\right\|^{2}-\varepsilon & \left\|P\left(u_{n}, v_{n}\right)\right\| \\
& \leq\left|-\left\|P\left(u_{n}, v_{n}\right)\right\|^{2}-\frac{1}{2}\left\langle\varphi^{\prime}\left(u_{n}, v_{n}\right),\left(u_{n}-v_{n}, v_{n}-u_{n}\right)\right\rangle\right| \\
& \leq k\left\|P\left(u_{n}, v_{n}\right)\right\|\left(\left\|u_{n}\right\|_{L^{2^{*}}}^{2^{*}-1}+\left\|v_{n}\right\|_{L^{2^{*}}}^{2^{*}-1}\right) .
\end{aligned}
$$

Therefore,

$$
\begin{aligned}
& \left\|Q\left(u_{n}, v_{n}\right)\right\|-\varepsilon \leq k\left(\left\|u_{n}\right\|_{L^{2^{*}}}^{2^{*}-1}+\left\|v_{n}\right\|_{L^{2^{*}}}^{2^{*}-1}\right) \\
& \left\|P\left(u_{n}, v_{n}\right)\right\|-\varepsilon \leq k\left(\left\|u_{n}\right\|_{L^{2^{*}}}^{2^{*}-1}+\left\|v_{n}\right\|_{L^{2^{*}}}^{2^{*}-1} .\right.
\end{aligned}
$$

Adding (3.10) and (3.11), and combining the result with (3.7) leads to

$$
\left\|\left(u_{n}, v_{n}\right)\right\|-2 \varepsilon \leq c_{1}+c_{2}\left\|\left(u_{n}, v_{n}\right)\right\|^{\left(2^{*}-1\right) / 2^{*}} ;
$$

so, $\left\{\left(u_{n}, v_{n}\right)\right\}$ is bounded in $X$.

We are now ready to establish the existence of a solution to the problem (3.1).

Theorem 3.5. Problem (3.1) has a non trivial solution.

Proof. Take $\Omega=\mathbb{R}^{N} \backslash \mathbb{Z}^{N}$. By lemma 3.4, there exists a bounded sequence $\left(u_{n}, v_{n}\right) \subset X$ satisfying (3.6)

Now, let us assume that:

$$
\begin{aligned}
& \delta_{1}:=\varlimsup_{n \rightarrow \infty} \sup _{a \in \mathbb{Z}^{N}} \int_{B(a, \sqrt{N})}\left|u_{n}\right|^{2^{*}}=0 \\
& \delta_{2}:=\varlimsup_{n \rightarrow \infty} \sup _{a \in \mathbb{Z}^{N}} \int_{B(a, \sqrt{N})}\left|v_{n}\right|^{2^{*}}=0
\end{aligned}
$$

then Lemma 2.3 implies that $u_{n}, v_{n} \rightarrow 0$ in $L^{2^{*}}\left(\mathbb{R}^{N}\right)$. This fact and (3.7) lead to a contradiction since $c>0$.

Therefore, we must have $\delta:=\max \left\{\delta_{1}, \delta_{2}\right\}>0$. Taking a subsequence if necessary, we may assume the existence of $a_{n} \in \mathbb{Z}^{N}$ such that

$$
\int_{B\left(a_{n}, \sqrt{N}\right)}\left|u_{n}\right|^{2^{*}}+\left|v_{n}\right|^{2^{*}} d x>\frac{\delta}{2} .
$$

The sequence $\left(\hat{u}_{n}, \hat{v}_{n}\right)$ defined by $\hat{u}_{n}(x):=u_{n}\left(x+a_{n}\right)$ and $\hat{v}_{n}(x):=v_{n}\left(x+a_{n}\right)$ is such that

$$
\int_{B(0, \sqrt{N})}\left|\hat{u}_{n}\right|^{2^{*}}+\left|\hat{v}_{n}\right|^{2^{*}} d x>\frac{\delta}{2}
$$

and satisfies (3.6) by $\mathbb{Z}^{N}$ invariance. Taking again a subsequence, if needed, we may assume that

$$
\left(\hat{u}_{n}, \hat{v}_{n}\right) \rightarrow(u, v) \text { in } X .
$$


Since $\hat{u}_{n} \rightarrow u, \hat{v}_{n} \rightarrow v$ in $L_{l o c}^{2}\left(\mathbb{R}^{N}\right)$, then $(u, v) \neq 0$. Finally, the weakly sequentially continuity of $\varphi^{\prime}$ gives

$$
\left\|\varphi^{\prime}(u, v)\right\| \leq \liminf _{n \rightarrow \infty}\left\|\varphi^{\prime}\left(\hat{u}_{n}, \hat{v}_{n}\right)\right\|=0 .
$$

Consequently $(u, v)$ is a non trivial solution of (3.1).

Now, we will study the following problem in $\Omega:=\mathbb{R}^{l} \times \omega$ a cylindrical domain with $\omega$ a bounded domain of $\mathbb{R}^{N-l}$ with for $1 \leq l \leq N-1$ :

$$
\left\{\begin{array}{l}
-\Delta u=\gamma v+|v|^{2^{*}-2} v \\
-\Delta v=\lambda u+|u|^{2^{*}-2} u \\
u, v \in H_{0}^{1}(\Omega)
\end{array}\right.
$$

where $0<\gamma, \lambda<\lambda_{1}(\Omega)$, with $\lambda_{1}(\Omega)$ the best constant in the Poincaré inequality,

$$
\lambda_{1}(\Omega):=\inf \left\{\int_{\Omega}|\nabla u|^{2} d x: u \in H_{0}^{1}(\Omega), \int_{\Omega} u^{2} d x=1\right\} .
$$

It is shown in [5] that $\lambda_{1}(\Omega)>0$.

The Poincaré inequality implies that the usual norm on $X:=H_{0}^{1}(\Omega) \times H_{0}^{1}(\Omega)$ is equivalent to the norm induced by the scalar product defined in (3.2). If we set $Y$ and $Z$ as in (3.3), and we denote by $P$ (resp. $Q$ ) the projection of $X$ onto $Y$ (resp. $Z$ ), the problem (3.12) allows a variationnal formulation since its solutions are critical points of the functional $\varphi_{1}: X \rightarrow \mathbb{R}$ defined by

$$
\begin{aligned}
\varphi_{1}(u, v) & :=\int_{\Omega} \nabla u \cdot \nabla v-\frac{\gamma u^{2}}{2}-\frac{\lambda v^{2}}{2}-\frac{|u|^{2^{*}}}{2^{*}}-\frac{|v|^{2^{*}}}{2^{*}} d x \\
& =\frac{\|Q(u, v)\|^{2}}{2}-\frac{\|P(u, v)\|^{2}}{2}-\psi(u, v)-\hat{\psi}(u, v) \\
& =\varphi(u, v)-\hat{\psi}(u, v),
\end{aligned}
$$

where

$$
\hat{\psi}(u, v):=\int_{\Omega} \gamma \frac{u^{2}}{2}+\lambda \frac{v^{2}}{2} d x
$$

and where $\varphi$ and $\psi$ are defined at the beginning of this section. It is easy to see that $\hat{\psi}$ is $C^{1}$, weakly sequentially lower semi-continuous, and $\hat{\psi}^{\prime}$ is weakly sequentially continuous. Also,

$$
\left\langle\hat{\psi}^{\prime}(u, v),(w, z)\right\rangle=\int_{\Omega} \gamma u w+\lambda v z d x .
$$

The follwing result establishes the existence of a bounded Palais-Smale sequence of $\varphi_{1}$. However, in writing $x:=(y, z)$, where $y \in \mathbb{R}^{l}$ and $z \in \omega$, we observe that for each $(u, v) \in X$ and each $a \in \mathbb{R}^{l}$

$$
\varphi_{1}(u, v)=\varphi_{1}(\hat{u}, \hat{v})
$$

where $\hat{u}:=u(y+a, z), \hat{v}:=v(y+a, z)$. So $\varphi_{1}$ is invariant under $\mathbb{R}^{l}$-translations and consequently, the Palais-Smale condition fails at all critical levels.

Lemma 3.6. There exists $c>0$ and a bounded sequence $\left\{\left(u_{n}, v_{n}\right)\right\}$ in $X$ such that

$$
\varphi_{1}\left(u_{n}, v_{n}\right) \rightarrow c>0, \quad \varphi_{1}^{\prime}\left(u_{n}, v_{n}\right) \rightarrow 0
$$

Proof. Set $\beta:=(\gamma+\lambda) / 2$. In using the Poincaré inequality and the Sobolev embedding Theorem, it follows the existence of a constant $C \geq 0$ such that for every $(u, u) \in Z$

$$
\varphi_{1}(u, u) \geq\left(\frac{\lambda_{1}(\Omega)-\beta}{2 \lambda_{1}(\Omega)}\right)\|(u, u)\|^{2}-C\|(u, u)\|^{2^{*}} .
$$

Therefore, there exists $r>0$ such that

$$
\inf _{\substack{(u, u) \in Z \\\|(u, u)\|=r}} \varphi_{1}(u, u)>0=\min _{\substack{(u, u) \in Z \\\|(u, u)\| \leq r}} \varphi_{1}(u, u) .
$$


The fact that for $(u, v) \in X$

$$
\varphi_{1}(u, v) \leq \varphi(u, v)
$$

allows us to use the arguments contained in the proof of Lemma 3.3 in order to show that there exists $\rho>r$ such that

$$
\max _{M_{0}} \varphi_{1}=0 \text { and } \sup _{M} \varphi_{1}<\infty
$$

where $M$ and $M_{0}$ are given respectively by (1.1) and (1.2).

Theorem 1.1 ensures the existence of a sequence $\left\{\left(u_{n}, v_{n}\right)\right\}$ in $X$ satisfying (3.13) for some $c>0$. It remains to show that the sequence is bounded. Let us observe that

$$
\varphi_{1}\left(u_{n}, v_{n}\right)-\frac{1}{2}\left\langle\varphi_{1}^{\prime}\left(u_{n}, v_{n}\right),\left(u_{n}, v_{n}\right)\right\rangle=\varphi\left(u_{n}, v_{n}\right)-\frac{1}{2}\left\langle\varphi^{\prime}\left(u_{n}, v_{n}\right),\left(u_{n}, v_{n}\right)\right\rangle,
$$

which implies (3.7).

Without lost of generality, we may assume that $\lambda \geq \gamma$. A straightforward computation leads to

$$
\begin{aligned}
\frac{1}{2}\left\langle\hat{\psi}^{\prime}\left(u_{n}, v_{n}\right),\left(u_{n}+v_{n}, u_{n}+v_{n}\right)\right\rangle & \leq \frac{\lambda}{2}\left\|u_{n}+v_{n}\right\|_{L^{2}}^{2} \\
& \leq \frac{\lambda}{2 \lambda_{1}(\Omega)}\left\|\nabla\left(u_{n}+v_{n}\right)\right\|_{L^{2}}^{2} \\
& =\frac{\lambda}{4 \lambda_{1}(\Omega)}\left\|\left(u_{n}+v_{n}, u_{n}+v_{n}\right)\right\|^{2} \\
& =\frac{\lambda}{\lambda_{1}(\Omega)}\left\|Q\left(u_{n}, v_{n}\right)\right\|^{2} .
\end{aligned}
$$

Similarly, we have

$$
\frac{1}{2}\left\langle\hat{\psi}^{\prime}\left(u_{n}, v_{n}\right),\left(u_{n}-v_{n}, v_{n}-u_{n}\right)\right\rangle \leq \frac{\lambda}{\lambda_{1}(\Omega)}\left\|P\left(u_{n}, v_{n}\right)\right\|^{2} .
$$

In combining respectively the last two equations with (3.8) and (3.9), the inequalities (3.10) and (3.11) become respectively:

$$
\begin{aligned}
& \left(\frac{\lambda_{1}(\Omega)-\lambda}{\lambda_{1}(\Omega)}\right)\left\|Q\left(u_{n}, v_{n}\right)\right\|-\varepsilon \leq k\left(\left\|u_{n}\right\|_{L^{2^{*}}}^{2^{*}-1}+\left\|v_{n}\right\|_{L^{2^{*}}}^{2^{*}-1}\right) \\
& \left(\frac{\lambda_{1}(\Omega)-\lambda}{\lambda_{1}(\Omega)}\right)\left\|P\left(u_{n}, v_{n}\right)\right\|-\varepsilon \leq k\left(\left\|u_{n}\right\|_{L^{2^{*}}}^{2^{*}-1}+\left\|v_{n}\right\|_{L^{2^{*}}}^{2^{*}-1}\right)
\end{aligned}
$$

and the conclusion follows as in the proof of Lemma 3.4.

We are now ready to establish the existence of a solution to the problem (3.12).

Theorem 3.7. If $\gamma, \lambda \in] 0, \lambda_{1}(\Omega)[$, then Problem (3.12) has a non trivial solution.

Proof. Let $\left(u_{n}, v_{n}\right) \subset X$ be a bounded sequence given in Lemma 3.6, and define

$$
\begin{aligned}
\delta_{1} & :=\varlimsup_{n \rightarrow \infty} \sup _{x \in \Omega} \int_{B(x, 1)}\left|u_{n}\right|^{2^{*}}, \\
\delta_{2} & :=\varlimsup_{n \rightarrow \infty} \sup _{x \in \Omega} \int_{B(x, 1)}\left|v_{n}\right|^{2^{*}} .
\end{aligned}
$$

In arguing as in the proof of Theorem 3.5 with Lemma 2.2, we show that $\delta:=\max \left\{\delta_{1}, \delta_{2}\right\}>0$, and hence we deduce the existence of a sequence $x_{n}=\left(y_{n}, z_{n}\right) \in \Omega$ such that

$$
\int_{B\left(x_{n}, 1\right)}\left|u_{n}\right|^{2^{*}}+\left|v_{n}\right|^{2^{*}} d x>\frac{\delta}{2}
$$

The sequence $\left(\hat{u}_{n}, \hat{v}_{n}\right)$ defined by $\hat{u}_{n}(x):=u_{n}\left(y+y_{n}, z\right)$ and $\hat{v}_{n}(x):=v_{n}\left(y+y_{n}, z\right)$ is such that

$$
\int_{B\left(\left(0, z_{n}\right), 1\right)}\left|\hat{u}_{n}\right|^{2^{*}}+\left|\hat{v}_{n}\right|^{2^{*}} d x>\frac{\delta}{2}
$$


and satisfies (3.13) by $\mathbb{R}^{l}$ invariance. By letting $R:=\max _{z \in \omega}|z|$, we obtain

$$
\int_{B(0, R+1)}\left|\hat{u}_{n}\right|^{2^{*}}+\left|\hat{v}_{n}\right|^{2^{*}} d x>\frac{\delta}{2}
$$

Finally, taking a subsequence, if needed, we may assume that

$$
\left(\hat{u}_{n}, \hat{v}_{n}\right) \rightarrow(u, v) \text { in } X
$$

As in the proof of Theorem 3.5, we conclude that $(u, v)$ is a non trivial solution of (3.12).

Acknowledgement. The first author wants to thank Marlène Frigon and the Department of Mathematics and Statistic of the University of Montréal for the hospitality he has received during his postdoctoral studies.

\section{References}

[1] V. Benci, and P.H. Rabinowitz, Critical point theorems for indefinite functionals, Invent. Math. 52 (1979), $241-273$.

[2] F. Clarke, and I. Ekeland, Hamiltonian trajectories having prescribed minimal period, Comm. Pure Appl. Math. 33 (1980), 103-116.

[3] F. Colin, "Inégalités de Hardy sur des domaines non bornés", Thèse de docotorat (Ph.D.), Université de Sherbrooke, Sherbrooke, 2002.

[4] F. Colin, Lemmes de décomposition appliqués à des inégalités de type Hardy-Sobolev, submitted to Potential Analysis.

[5] M.J. Esteban, Nonlinear elliptic problems in strip-like domains: symmetry of positive vortex rings, Nonlinear Anal. 7 (1983), 365-379.

[6] P. Felmer, and Z.Q. Wang, Multiplicity for symmetric indefinite functionals: application to Hamiltonian and elliptic systems, Topol. Methods Nonlinear Anal. 12 (1998), 207-226.

[7] J. Hulshof, and R. van der Vorst, Differential systems with strongly indefinite variational structure, J. Funct. Anal. 114 (1993), 32-58.

[8] J. Hulshof, E. Mitidieri and R. van der Vorst, Strongly indefinite systems with critical Sobolev exponents, Trans. Amer. Math. Soc. 350 (1998), 2349-2365.

[9] W. Kryszewski, and A. Szulkin, Generalized linking theorem with an application to a semilinear Schrödinger equation, Adv. Differential Equations 3 (1998), 441-472.

[10] P.-L. Lions, The concentration-compactness principle in the calculus of variations. The locally compact case, part 2, Ann. Inst. Henri-Poincaré 1 (1984), 223-283.

[11] M. Ramos, Z.-Q. Wang, and M. Willem, Positive solutions for elliptic equations with critical growth in unbounded domains, in "Calculus of variations and differential equations, Technion 1988", Chapman and Hall, Boca Raton, 1999, 192-199.

[12] Z.-Q. Wang, and M. Willem, Singular minimization problems, J. Differential Equations 161 (2000), 307-320.

[13] M. Willem, "Minimax Theorems", Birkhauser, Boston, 1996. 